\title{
Article \\ Ethyl loflazepate as a treatment for patients with idiopathic and psychogenic taste disorder
}

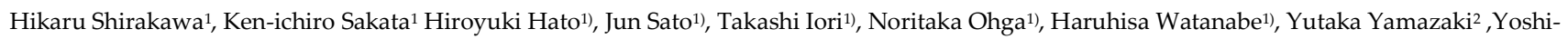
masa Kitagawa $\left.{ }^{1}\right)$ Corresponding author

\author{
Department of Oral Diagnosis and Medicine, Division of Oral Pathobiological Science, Faculty of Dental Medi- \\ cine and Graduate School of Dental Medicine, Hokkaido University. (Chief: Prof. Yoshimasa Kitagawa) \\ Kita-13 Nishi-7, Kita-ku, Sapporo 060-8586, Hokkaido, Japan \\ Department of Gerodontology, Division of Oral Health Science, Faculty of Dental Medicine and Graduate \\ School of Dental Medicine, Hokkaido University. (Chief: Prof. Yutaka Yamazaki) Kita-13 Nishi-7, Kita-ku, \\ Sapporo 060-8586, Hokkaido, Japan
}

${ }^{*}$ Corresponding author

Ken-ichiro Sakata, DDS, PhD

Department of Oral Diagnosis and Medicine, Division of Oral Pathobiological Science, Faculty of Dental Medicine and Graduate School of Dental Medicine, Hokkaido University. (Chief: Prof. Yoshimasa Kitagawa)

Kita-13 Nishi-7, Kita-ku, Sapporo 060-8586, Hokkaido, Japan

Phone/Fax: +81-11-706-4280 E-mail: sakata-0303@den.hokudai.ac.jp

\begin{abstract}
Ethyl loflazepate (EL) is a benzodiazepine derivative that has been reported to activate the gustatory cortex. Our department routinely uses EL as a first-line treatment for idiopathic and psychogenic taste disorders, although little has been reported in the literature with respect to patient outcomes, so we conducted a retrospective study examining its safety and efficacy. Between 2008 and 2020, 49 patients (14 males and 35 females; mean age, 62.1 years) were diagnosed with taste disorders and received EL as their only treatment for $>14$ days. Severity of taste disorder was evaluated using the paper disc method by Sakai et al [7], and treatment efficacy was evaluated using the Visual Analogue Scale, wherein patients gave subjective ratings for their symptoms (reductions by $>50 \%$ after administration of EL for 4 weeks were defined as mprovements). Results showed that the improvement rates for patients with idiopathic and psychogenic taste disorders were $55 \%$ and $70 \%$, respectively. Additionally, the majority (78\%) improved within 2 weeks, and side effects were mild (seven cases of sleepiness and one case of dizziness). We conclude that EL is an appropriate first-line medication for patients with idiopathic and psychogenic taste disorders.
\end{abstract}

Keywords: ethyl loflazepate, idiopathic taste disorder, psychogenic taste disorder

\section{Introduction}

Ethyl loflazepate (EL) is a benzodiazepine derivative that is safe for elderly individuals and is well documented to treat anxiety and dental psychosomatic disorders [1-3]. Note worthily within the last two decades, research has also reported that this drug activates the gustatory cortex [4]. Our department systematically diagnoses and treats taste disorders [5], and we frequently employ EL as a first-line therapy when they are suspected of arising spontaneously or having a psychological origin. At present, few investigations have evaluated outcomes following the administration of EL in patients with idiopathic or psychogenic taste disorders [6], so we conducted a retrospective study examining its safety and efficacy among cases presented to our team. 


\section{Materials and Methods}

\subsection{Participants}

Between January 2008 and January 2019, patients complaining of gustatory dysfunction were admitted to the Department of Oral Medicine at Hokkaido University Hospital Dental Center and treated with EL. In total, the safety of EL was assessed in 55 patients, although 6 dropped out early because of side effects. This rendered a final number of 49 patients [14 males and 35 females; age, 62.1 years (range, 29×84 years); and average duration of the disease, 25 months] for efficacy analysis, given that they received the drug for $>2$ weeks (Figure 1 and Table 1).

With regard to efficacy analysis, EL was a first-line therapy for 42 patients and a second-line therapy for 7 patients who were previously treated for oral candida with the antifungal drug Flored gel $^{\circledR}$, yet they experienced no improvements in taste. Upon examination, 22 and 27 patients were diagnosed as having an idiopathic or psychogenic taste disorder, respectively, per the criteria listed in Figure 1. Of note, the former has no established etiology (Table1), whereas the latter is considered to have a psychological basis according to various tests (e.g., CMI, SDS, and PHQ-9). Subjective symptoms of taste disorders were judged according to the definition shown in Table2 [5].

This study was conducted with the approval of the Hokkaido University Hospital Independent Clinical Research Review Committee (Approval No. 018-0381: Clinical study in patients with taste disorders.). All eligible patients gave their informed consent for inclusion before they participated in the study. The study was conducted in accordance with the Declaration of Helsinki.

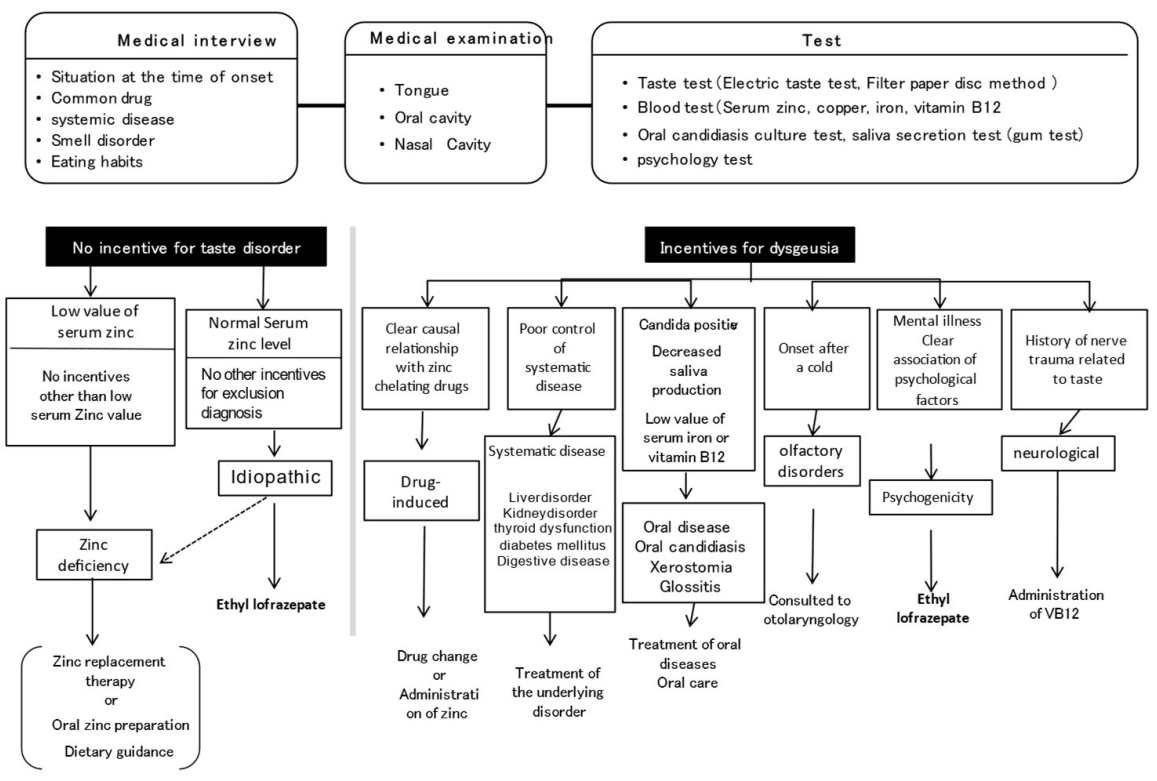

Figure1. Diagnosis and treatment of taste disorders 
- Zinc deficiency: Serum zinc level is less than $70 \mu \mathrm{g} / \mathrm{dl}$ and there are no other obvious causes.

- Idiopathic: There are no other obvious causes of taste disorder, excluding psychogenicity.

- Oral disease: Those with taste disorders closely related to oral diseases such as candidiasis, xerostomia, glossitis and tongue coating

- Psycogenic:Psychological factors clearly related to onset and progress of the symptoms.

- Systematic disease: Currently, the state of control of systemic disease is poor (Liver disorder, renal disorder, diabetes, gastrointestinal disorder).

- Drug induced:There is described in the side effects information, and it is clear causal relationship

- Post commmon cold: Aware of the taste disorders after the common cold

- olfactory disorders: Simultaneous disorder of taste disorder and olfactory disorder,and taste is normal, but it involves only olfaction disorder.

- Latrogenic: Those that developed after middle ear surgery or laryngeal microsurgery and palatine tonsil surgery

Table 1:Taste disorders are caused by several factors

Phantogeusia:It has a certain taste even though there is nothing in the mouth

Hypogeusia: The taste of food becomes lighter

Taste hypersensitivity: The taste of food becomes strong

Defferent taste:Feel the taste different from the original taste

Dissociable: You don't feel only a specific taste

Bad taste: It tastes bad no matter what you eat

Table 2 :Subjective symptoms of taste disorders

\subsection{Clinical examination}

Severity of taste disorder was evaluated using Taste Discs ${ }^{\circledR}$ (Sanwa Kagaku, Nagoya) and the method outlined by Sakai et al. [7]. Scores for perception of sweetness, saltiness, sourness, and bitterness in the tympanolar region that were $<3.5,3.5-4.5,4.5-5.5$, and $>5.5$ were recognized as normal taste function, mild taste disorder, moderate taste disorder, and severe taste disorder, respectively. Measurement of serum zinc levels was performed using an atomic fluorescence analyzer (AA240FS, Agilent, Santa Clara, CA, USA), with normal values ranging from 70 to $111 \mu \mathrm{g} / \mathrm{dL}$ according to Harrison's Principles of Internal Medicine [9].

\subsection{Administration of EL}

One tablet (1 mg) of EL was administered before going to bed at night. If a patient experienced daytime sleepiness or heart flutters, the dosage was reduced to $0.5 \mathrm{mg}$.

\subsection{Evaluation of efficacy}

Treatment efficacy of EL was evaluated using the Visual Analogue Scale (VAS) [10]. Patients who exhibited improvements after 4 weeks were defined as those with $>50 \%$ decrease in subjective symptoms, and those regarded as unchanged had $<50 \%$ decrease in subjective symptoms. 
In two cases, no improvement in patients' conditions was detected after 2 weeks, so administration of EL was discontinued, and patients were given another agent for a duration of 2-4 weeks. Consequently, these individuals were only evaluated at the time when they decided to discontinue EL treatment.

\subsection{Statistical analysis}

Improvement rates according to age, gender, duration of the disease, subjective symptoms, cause of dysgeusia, period of EL administration, length of time to experience an effect from EL, serum zinc values, and severity of taste disorder were compared using Fisher's exact test, and $\mathrm{p}<0.05$ was considered significant. The type and incidence of adverse events were examined as a means to determine safety.

\section{Results}

Among the 55 patients assessed for treatment safety, 6 dropped out because of sleepiness and 1 dropped out because of urticaria. Shortly after discontinuing EL administration, these negative effects lessened or abated in the five cases (Figure 2).

Out of the 49 patients evaluated for treatment efficacy, phantogeusia was observed in 26 cases, hypogeusia in 10 cases, and complaints of different taste in 7 cases. In addition, 31 patients displayed an improvement in taste disorder (i.e., rate of 63\%), 18 remained unchanged (i.e., rate of $37 \%$ ), and no one experienced worsening of their condition. Moreover, no significant differences in improvement rates for the indices delineated above were observed (Table 2). Adverse events of drowsiness or dizziness were reported by seven patients and one patient, respectively, although all symptoms were minor, so administration of EL continued. For two patients, side effects from EL were slightly more intense, so the drug's dosage was reduced to $0.5 \mathrm{mg}$.

Across all patients who regained at least some taste function, they were able to cease treatment after maintaining a consistent dosage of EL over the course of 2-3 months.

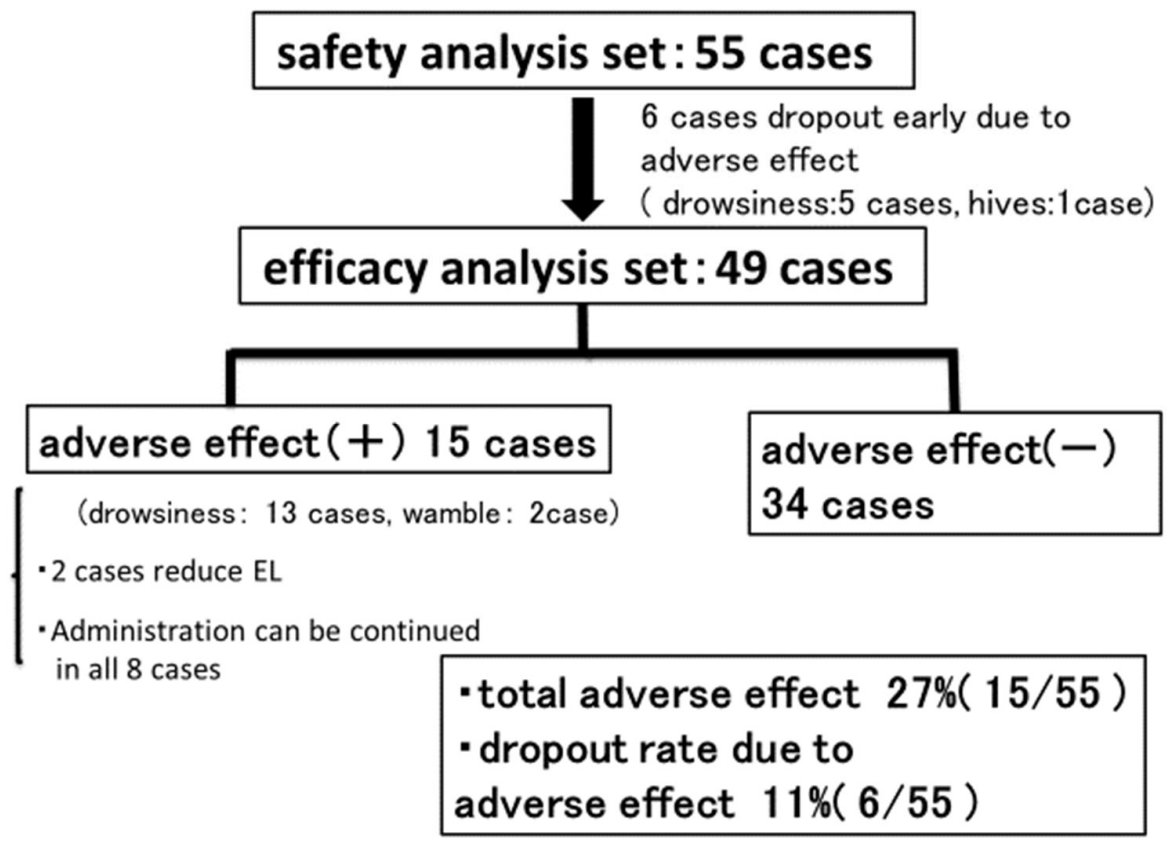

Figure2:Profile of this study 


\begin{tabular}{|c|c|c|c|}
\hline & & Improve rate & \\
\hline Age(median:65 years) & $\begin{array}{c}65 \text { years of age or older } \\
\text { Under } 65\end{array}$ & $\left.\begin{array}{c}63 \%(17 / 27) \\
64 \%(14 / 22)\end{array}\right]$ & n.s. \\
\hline Sex & $\begin{array}{c}\text { Man } \\
\text { Woman }\end{array}$ & $\left.\begin{array}{ll}71 \% & (10 / 14) \\
60 \% & (21 / 35)\end{array}\right]$ & n.s. \\
\hline Desease period & $\begin{array}{c}\text { Over } 1 \text { year } \\
\text { Less than a year }\end{array}$ & $\left.\begin{array}{cc}58 \% & (11 / 19) \\
67 \% & (20 / 30)\end{array}\right]$ & n.s. \\
\hline Subjective symptoms of taste & $\begin{array}{l}\text { Self-motivated } \\
\text { Hypogeusia } \\
\text { Different taste }\end{array}$ & $\left.\begin{array}{c}65 \%(17 / 26) \\
80 \%(8 / 10) \\
57 \%(4 / 7)\end{array}\right]$ & n.s. \\
\hline Causes & $\begin{array}{l}\text { Psychogenic } \\
\text { Idiopathic }\end{array}$ & $\left.\begin{array}{c}70 \%(19 / 27) \\
55 \%(12 / 22)\end{array}\right]$ & n.s. \\
\hline Dosing time & $\begin{array}{c}\text { First dose } \\
\text { After administration of other drug }\end{array}$ & $\left.\begin{array}{ll}62 \% & (26 / 42) \\
71 \% & (5 / 7)\end{array}\right]$ & n.s. \\
\hline $\begin{array}{l}\text { Period until the effect appears } \\
※ 19 \text { effective cases out of } 31 \\
\text { cases }\end{array}$ & $\begin{array}{l}\sim 7 \text { day } \\
8 \sim 14 \text { day } \\
15 \sim 28 \text { day }\end{array}$ & $\left.\begin{array}{cc}39 \% & (12 / 31) \\
39 \% & (12 / 31) \\
22 \% & (7 / 31)\end{array}\right]$ & n.s. \\
\hline Filter paper disc method & $\begin{array}{c}\text { Normal } \\
\text { Mild } \\
\text { Moderate degree } \\
\text { Severe }\end{array}$ & $\left.\begin{array}{cc}71 \% & (12 / 17) \\
60 \% & (6 / 10) \\
72 \% & (8 / 11) \\
46 \% & (5 / 11)\end{array}\right]$ & n.s. \\
\hline
\end{tabular}

\section{Figure3:Results for effectiveness analysis}

\section{Discussion}

Causes of taste disorders are multitudinous and can be attributed to the common cold, zinc deficiency, olfactory dysfunction, and systemic, oral, iatrogenic, idiopathic, and psychogenic diseases [8]. Treatment for the majority of these issues is well established, but effective options for managing the last two remain to be identified [5]. Within the realm of otolaryngology, zinc replacement therapy has traditionally been recommended for individuals with idiopathic and psychogenic dysgeusia [11-13], which is why our department has actively administered polaprezinc. Unfortunately, improvement rates have not exceeded $22 \%$.

Against this background, one contemporary investigation by Takahashi et al[1]. reported that EL is successful at correcting or improving abnormal taste function emerging spontaneously, and another by Shimura et al[4]. found that EL specifically stimulates the gustatory cortex. Moreover, unlike benzodiazepine drugs and polabrezinc [16,17, 18, 19], EL is known to act in a short time (i.e., half-life is $122 \mathrm{~h}$ ) and for ultra-long, prevents or treats anxiety, and appears to exert minimal side effects (e.g., memory loss and drowsiness) or to possess addictive qualities $[14,15]$. Thus, we believed that EL would be an ideal therapy for the cases presented to our hospital.

In this study, there was an overall improvement rate in taste disorders of $63 \%(31 / 49)$ : $55 \%(12 / 22)$ for patients with idiopathic dysgeusia and 70\%(19/27 for patients with psychogenic dysgeusia. In addition, 78\%(12/31) of those for whom EL was effective experienced improvement within 2 weeks of administration. Surprisingly, no significant differences in improvement rates by age, gender, duration of the disease, subjective symptoms, cause of dysgeusia, period of EL administration, length of time to experience an effect from EL, serum zinc values, or severity of taste disorder were observed. Prior research, in particular, has demonstrated that upon treatment, the duration of a disease decreases significantly after $\geq 6$ months and it takes a while for patients to experience improvements [11], yet for our cohort, there was no significant difference in improvement rates between patients with a course of $>1$ year vs. $<1$ year. This finding bears witness to the expediency of EL over other medications commonly employed in such instances. 
Concerning lack of improvement or side effects, the incidence rate was $27 \%(15 / 49)$. Five patients chose to discontinue EL treatment in favor of different drugs because they experienced no alteration in their condition, and the rest predominantly had mild sleepiness. Written on the medical packaging for EL are the main side effects, including psychiatric disorders (e.g., drowsiness and reduced cognition and concentration) and central and peripheral nervous system disorders (e.g., stabilization and speech problems, and head sensations), and a minor side effect, that is, loss of taste. Importantly for the latter, the frequency of loss of taste is described as $<0.1 \%$, but we could not locate evidence of this phenomenon in the literature nor did anyone in our cohort complain of this. A mere amount of $1 \mathrm{mg}$ of EL was administered to our patients because the drug's manufacturers state that the daily dosage for insomnia is $2 \mathrm{mg}$. Hence, this would likely induce strong sleepiness. Going forward, healthcare professionals should be diligent in informing their patients regarding the aforementioned side effects and monitoring them carefully after EL administration.

\section{Conclusions}

This study examined the safety and efficacy of EL in patients with idiopathic and psychogenic taste disorders between 2008 and 2020 and found that it resulted in an overall improvement rate of $63 \%(31 / 49)$. In particular, among the 24 patients for whom EL was effective, $78 \%(24 / 31)$ experienced improvements within 2 weeks and an improvement rate of $50 \%$ was observed when the disease had lasted a year or longer. In addition, adverse reactions occurred in 13 patients, with the most common being sleepiness. We conclude that EL monotherapy could be given as a first-line treatment for patients with idiopathic and psychogenic taste disorders.

6. Author Contributions: Ken-Ichiro,S. and Yutaka,Y. conceived of and designed the study. .Ken-

Ichiro,S, Hiroyuki,Hato,Takashi Iori,Jun,Sato, Noritaka,Ohga and Haruhisa,Watanabe devel oped the theory, collected the data, and performed the computations.Hikaru,S and Ken-Ichiro,S verified the analytical methods. Yoshimasa,K encouraged Ken-Ichiro,S to investigate [a specific aspect] and supervised the findings of this work. 


\section{References}

1. Akira, T.; Naoki, I.; Masazumi, S.; Hiromasa, T.; Toshihiro, K. The efficacy of co-administration of fluvoxamine and loflazepate ethyl for oral psychosomatic patients. Jpn J Psychosom Dent 2005, 20, 50-54.

2. Paudel, D.; Utsunomiya, M.; Yoshida, K.; Giri, S.; Uehara, O.; Matsuoka, H.; Chiba, I.; Toyofuku, A.; Abiko, Y. Pharmacotherapy in relieving the symptoms of burning mouth syndrome: A 1-year follow-up study. Oral Dis 2020 , 26, 193-199. DOI: 10.1111/odi.13226

3. Heckmann, S. M.; Kirchner, E.; Grushka, M.; Wichmann, M. G.; Hummel, T. A double-blind study on clonazepam in patients with burning mouth syndrome. Laryngoscope 2012, 122, 813-816. DOI: 10.1002/lary.22490

4. Shimura, T.; Kamada, Y.; Yamamoto, T. Ventral tegmental lesions reduce overconsumption of normally preferred taste fluid in rats. Behav Brain Res 2002, 134, 123-130. DOI: 10.1016/s0166-4328(01)00461-2

5. Yutaka, Y. Ken-Ichiro S;Jun S;Manabu O;Hironobu H;Atsushi M;Yoshimasa K.Clinical study of 210 cases of taste disorder. J Jpn Stomatol Soc 2013, 62, 247-253.

6. Hiromasa, T.; Akira, T.; Naoki, I.; Masazumi, S.; Aki, M.; Toshihiro, K. Efficacy of ethyl loflazepate for phantogeusia - a case study. Japanese Journal of Psychosomatic Dentistry 2006, 21, 23-26.

7. Fumitaka, S.; Shinya, Y.; Sohei, E.; Hiroshi, T. Double-blind, pracebo controlled trial of zinc picolinate for taste disorders. Acta Oto-laryngol 2002, 546, 129-133.

8. Hiroshi, T.; Minoru, I.; Yukio, O. Basis and practice of clinical taste examination. Auris Nasus Larynx 1986, 13, 1-9

9. Paolo, M. S.; Robert, M. R. Harrison’s Principles Internal Medicine, 20th ed,;McGraw-Hill Education / Medical,ISBN 978-123-964-403-0, 2018.

10. McCormack, H. M.; Horne, D. J.; Sheather, S. Clinical applications of visual analogue scales;a critical review. Psycol Med 1988, 18, 1007-1019. DOI: 10.1017/s0033291700009934

11. Sakagami, M.; Ikeda, M.; Tomita, H.; Ikui, A.; Aiba, T.; Takeda, N.; Inokuchi, A.; Kurono, Y.; Nakashima, M.; Shibasaki, Y.; Yotsuya, O. A zinc-containing compound, Polaprezinc, is effective for patients with taste disorders:randomized, double-blind, placebocontrolled, multi-center study. Acta Oto-Laryngol 2009, 129, 1115-1120. DOI: $10.1080 / 00016480802552550$

12. Henkin, R. I.; Schecter, P. J.; Friedewald, W. T.; Demets, D. L.; Raff, M. A double blind study of the effects of zinc sulfate on taste and smell dysfunction. Am J Med Sci 1976, 272, 285-299. DOI: 10.1097/00000441-197611000-00006

13. Yoshida, S.; Endo, S.; Tomita, H. A double-blind study of the therapeutic efficacy of zinc gluconate on taste disorder. Auris Nasus Larynx 1991, 18, 153-161. DOI: 10.1016/s0385-8146(12)80219-7

14. Chambon, J. P.; Perio, A.; Demarne, H.; Hallot, A.; Dantzer, R.; Roncucci, R.; Bizière, K. Ethyl Loflazepate: A prodrug from the benzodiazepine series designed to dissociate anxiolytic and sedative activities. Arzneim Forsch 1985, 35, 1573-1577.

15. Goro, F. Tasuku H: yukihiko S; Tadashi H; Hiroyuki W; Mihisa F; Kenji H; Masaomi I. Effects of Etizolam and ethyl loflazepate on the P300 event-related potential in healthy subjects. Ann. Gen. Psychiatry 2010, 9, 37. DOI: $10.1186 / 1744-859 X-9-37$

16. Chouinard, G. Issues in the clinical use of benzodiazepines: Potency, withdrawal, and rebound. The Journal of Clinical J. Clin. Psychiatry 2004, 65, 7-12.

17. O'Brien, C. P. Benzodiazepine use, abuse, and dependence. J. Clin. Psychiatry 2005, 66, 28-33. DOI: 10.4088/JCP.v66n0104 
18. Ikeda, M.; Aiba, T.; Ikui, A.; Inokuchi, A.; Kurono, Y.; Sakagami, M.; Takeda, N.; Tomita, H. Taste of the examination methods and treatments used in Japan. Acta Oto-Laryngol 2005, 125, 1203-1210. DOI: 10.1080/00016480510040173

19. Akiko, S.; Tomomi, N.; Hideki, O.; Emi, M.; Atsushi, N.; Masanori, U.; Masafumi, S. Clinical analysis of 1059 patients with taste disorders. Nippon Jibiinkoka Gakkai Kaiho 2013, 116, 77-82. DOI: 10.3950/jibiinkoka.116.77 